\title{
About Robust Stability of Caputo Linear Fractional Dynamic Systems with Time Delays through Fixed Point Theory
}

\author{
M. De la Sen \\ Faculty of Science and Technology, University of the Basque Country, \\ 644 de Bilbao, Leioa, 48080 Bilbao, Spain \\ Correspondence should be addressed to M. De la Sen, manuel.delasen@ehu.es
}

Received 9 November 2010; Accepted 31 January 2011

Academic Editor: Marlène Frigon

Copyright (C) 2011 M. De la Sen. This is an open access article distributed under the Creative Commons Attribution License, which permits unrestricted use, distribution, and reproduction in any medium, provided the original work is properly cited.

\begin{abstract}
This paper investigates the global stability and the global asymptotic stability independent of the sizes of the delays of linear time-varying Caputo fractional dynamic systems of real fractional order possessing internal point delays. The investigation is performed via fixed point theory in a complete metric space by defining appropriate nonexpansive or contractive self-mappings from initial conditions to points of the state-trajectory solution. The existence of a unique fixed point leading to a globally asymptotically stable equilibrium point is investigated, in particular, under easily testable sufficiency-type stability conditions. The study is performed for both the uncontrolled case and the controlled case under a wide class of state feedback laws.
\end{abstract}

\section{Introduction}

Fractional calculus is concerned with the calculus of integrals and derivatives of any arbitrary real or complex orders. In this sense, it may be considered as a generalization of classical calculus which is included in the theory as a particular case. There is a good compendium of related results with examples and case studies in [1]. Also, there is an existing collection of results in the background literature concerning the exact and approximate solutions of fractional differential equations of Riemann-Liouville and Caputo types [1-4], fractional derivatives involving products of polynomials $[5,6]$, fractional derivatives and fractional powers of operators [7-9], boundary value problems concerning fractional calculus (see for instance $[1,10]$ ) and so forth. On the other hand, there is also an increasing interest in the recent mathematical related to dynamic fractional differential systems oriented towards several fields of science like physics, chemistry or control theory. Perhaps the reason of interest in fractional calculus is that the numerical value of the fraction parameter allows 
a closer characterization of eventual uncertainties present in the dynamic model. We can also find, in particular, abundant literature concerned with the development of Lagrangian and Hamiltonian formulations where the motion integrals are calculated though fractional calculus and also in related investigations concerned dynamic and damped and diffusive systems [11-17] as well as the characterization of impulsive responses or its use in applied optics related, for instance, to the formalism of fractional derivative Fourier plane filters (see, for instance, [16-18]), and Finance [19]. Fractional calculus is also of interest in control theory concerning for instance, heat transfer, lossless transmission lines, the use of discretizing devices supported by fractional calculus, and so forth (see, for instance [20-22]). In particular, there are several recent applications of fractional calculus in the fields of filter design, circuit theory and robotics [21, 22], and signal processing [17]. Fortunately, there is an increasing mathematical literature currently available on fractional differ-integral calculus which can formally support successfully the investigations in other related disciplines.

This paper is concerned with the investigation of the solutions of time-invariant fractional differential dynamic systems [23, 24], involving point delays which leads to a formalism of a class of functional differential equations, [25-31]. Functional equations involving point delays are a crucial mathematical tool to investigate real process where delays appear in a natural way like, for instance, transportation problems, war and peace problems, or biological and medical processes. The main interest of this paper is concerned with the positivity and stability of solutions independent of the sizes of the delays and also being independent of eventual coincidence of some values of delays if those ones are, in particular, multiple related to the associate matrices of dynamics. Most of the results are centred in characterizations via Caputo fractional differentiation although some extensions presented are concerned with the classical Riemann-Liouville differ-integration. It is proved that the existence nonnegative solutions independent of the sizes of the delays and the stability properties of linear time-invariant fractional dynamic differential systems subject to point delays may be characterized with sets of precise mathematical results.

On the other hand, fixed point theory is a very powerful mathematical tool to be used in many applications where stability knowledge is needed. For instance, the concepts of contractive, weak contractive, asymptotic contractive and nonexpansive mappings have been investigated in detail in many papers from several decades ago (see, for instance, [32-34] and references therein). It has been found, for instance, that contractivity, weak contractivity and asymptotic contractivity ensure the existence of a unique fixed pointing complete metric or Banach spaces. Some theory and applications of some types of functional equations in the context of fixed point theory have been investigated in [35, 36]. Fixed point theory has also been employed successfully in stability problems of dynamic systems such as time-delay and continuous-time/digital hybrid systems and in those involving switches among different parameterizations. This paper is concerned with the investigation of fixed points in Caputo linear fractional dynamic systems of real order $\alpha$ which involved delayed dynamics subject to a finite set of bounded point delays which can be of arbitrary sizes. The self-mapping defined in the state space from initial conditions to points of the state-trajectory solution are characterized either as nonexpansive or as contractive. The first case allows to establish global stability results while the second one characterizes global asymptotic stability.

\subsection{Notation}

$\mathbf{C}, \mathbf{R}$, and $\mathbf{Z}$ are the sets of complex, real, and integer numbers, respectively. 
$\mathbf{R}_{+}$and $\mathbf{Z}_{+}$are the sets of positive real and integer numbers, respectively, $\mathbf{C}_{+}$is the set of complex numbers with positive real part. $\mathbf{Z}_{+} \cup\{0\}$.

$\mathbf{C}_{0+}:=\mathbf{C}_{+} \cup\{\mathbf{i} \omega: \boldsymbol{\omega} \in \mathbf{R}\}$, where $\mathbf{i}$ is the complex unity, $\mathbf{R}_{0+}:=\mathbf{R}_{+} \cup\{0\}$ and $\mathbf{Z}_{0+}:=$

$\mathbf{R}_{-}$and $\mathbf{Z}_{-}$are the sets of negative real and integer numbers, respectively; and $\mathbf{C}_{-}$is the set of complex numbers with negative real part.

$\mathbf{C}_{0-}:=\mathbf{C}_{-} \cup\{\mathbf{i} \omega: \omega \in \mathbf{R}\}$, where $\mathbf{i}$ is the complex unity, $\mathbf{R}_{0}:=\mathbf{R}_{-} \cup\{0\}$ and $\mathbf{Z}_{0-}:=\mathbf{Z}_{-} \cup\{0\}$.

$\bar{N}:=\{1,2, \ldots, N\} \subset \mathbf{Z}_{0+}, " \vee$ " is the logic disjunction, and " $\wedge$ " is the logic conjunction. $[t / h]$ is the integer part of the rational quotient $t / h$.

$\sigma(M)$ denotes the spectrum of the real or complex square matrix $M$ (i.e., its set of distinct eigenvalues).

$\|\quad\|$ denotes any vector or induced matrix norm. Also, $\|m\|_{p}$ and $\|M\|_{p}$ are the $\ell_{p}$-norms of the vector $m$ or (induced) real or complex matrix $M$, and $\mu_{p}(M)$ denote the $\ell_{p}$ measure of the square matrix $M$, [20]. The matrix measure $\mu_{p}(M)$ is defined as the existing limit $\mu_{p}(M):=\lim _{\varepsilon \rightarrow 0^{+}}\left(\left\|I_{n}+\varepsilon X\right\|_{p}-\varepsilon\right) / \varepsilon$ which has the property $\max \left(-\|M\|_{p}, \max _{i \in \bar{n}} \operatorname{re} \lambda_{i}(M)\right) \leq \mu_{p}(M) \leq\|M\|_{p}$ for any square $n$-matrix $M$ of spectrum $\sigma(M)=\left\{\lambda_{i}(M) \in \mathbf{C}: 1 \leq i \leq n\right\}$. An important property for the investigation of this paper is that $\mu_{2}(M)<0$ if $M$ is a stability matrix: that is, if $\lambda_{i}(M)<0 ; 1 \leq i \leq n$.

$\|\quad\|_{\infty}$ denotes the supremum norm on $\mathbf{R}_{0+}$, or its induced supremum metric, for functions or vector and matrix functions without specification of any pointwise particular vector or matrix norm for each $t \in \mathbf{R}_{0+}$. If pointwise vector or matrix norms are specified, the corresponding particular supremum norms are defined by using an extra subscript. Thus, $\|m\|_{p_{\infty}}:=\sup _{t \in \mathbf{R}_{0}}\|m(t)\|_{p}$ and $\|M\|_{p \infty}:=\sup _{t \in \mathbf{R}_{0}}\|M(t)\|_{p}$ are, respectively, the supremum norms on $\mathbf{R}_{0+}$ for vector and matrix functions of domains in $\mathbf{R}_{0+} \times \mathbf{R}^{n}$, respectively, in $\mathbf{R}_{0+} \times \mathbf{R}^{n \times m}$ defined from their $\ell_{p}$ pointwise respective norms for each $t \in \mathbf{R}_{0+}$.

$I_{n}$ is the $n$th identity matrix.

$K_{p}(M)$ is the condition number of the matrix $M$ with respect to the $\ell_{p}$-norm;

$$
\bar{k}:=\{1,2, \ldots, k\} \text {. }
$$

The sets $\mathrm{BPC}^{(i)}\left(\right.$ dom, codom) and $\mathrm{PC}^{(i)}$ (dom, codom) are the sets of functions of a certain domain and codomain which are of class $C^{(i-1)}$ (dom, codom) and with the $i$ th derivative is bounded piecewise continuous, respectively, piecewise continuous in the definition domain.

\section{Caputo Fractional Linear Dynamic Systems with Point Constant Delays and the Contraction Mapping Theorem}

Consider the linear functional Caputo fractional dynamic system of order $\alpha$ with $r$ delays:

$$
\begin{aligned}
\left(D_{0+}^{\alpha} x\right)(t) & :=\frac{1}{\Gamma(k-\alpha)} \int_{0}^{t} \frac{x^{(k)}(\tau)}{(t-\tau)^{\alpha+1-k}} d \tau \\
& =\sum_{i=0}^{r} \widehat{A}_{i}(t) x\left(t-r_{i}\right)+B(t) u(t) \\
& =\sum_{i=0}^{r} A_{i} x\left(t-r_{i}\right)+\sum_{i=0}^{r} \tilde{A}_{i}(t) x\left(t-r_{i}\right)+B(t) u(t),
\end{aligned}
$$


with $k-1<\alpha\left(\in \mathbf{R}_{+}\right) \leq k ; k \in \mathbf{Z}_{+}, 0=r_{0}<r_{1}<r_{2}<\cdots<r_{r}=h<\infty$ being distinct constant delays, where $r_{i}(i \in \bar{r})$ are the $r$ (in general incommensurate delays) $0=r_{0}<r_{i}(i \in \bar{r})$ subject to the system piecewise continuous bounded matrix functions of delayed dynamics $\widehat{A}_{i}: \mathbf{R}_{0+} \rightarrow \mathbf{R}^{n \times n}(i \in \bar{r} \cup\{0\})$ which are decomposable as a (nonunique) sum of a constant matrix plus a bounded matrix function of time, that is, $\widehat{A}_{i}(t)=A_{i}+\widetilde{A}_{i}(t)$, for all $t \in \mathbf{R}_{0+}$, and $B: \mathbf{R}_{0+} \rightarrow \mathbf{R}^{n \times m}$ is the piecewise continuous bounded control matrix. The initial condition is given by $k n$-real vector functions $\varphi_{j}:[-h, 0] \rightarrow \mathbf{R}^{n}$, with $j \in \overline{k-1} \cup\{0\}$, which are absolutely continuous except eventually in a set of zero measure of $[-h, 0] \subset \mathbf{R}$ of bounded discontinuities with $\varphi_{j}(0)=x_{j}(0)=x^{(j)}(0)=x_{j 0}, j \in \overline{k-1} \cup\{0\}$. The function vector $u: \mathbf{R}_{0+} \rightarrow \mathbf{R}^{m}$ is any given bounded piecewise continuous control function. The following result is concerned with the unique solution on $\mathbf{R}_{0+}$ of the above differential fractional system (3.1). The proof, which is based on Picard-Lindelöf theorem, follows directly from a parallel existing result from the background literature on fractional differential systems by grouping all the additive forcing terms of (2.1) in a unique one (see for instance [1, (1.8.17), (3.1.34)(3.1.49)], with $\left.f(t) \equiv \sum_{i=1}^{r} A_{i} x\left(t-h_{i}\right)+B u(t)\right)$. For the sake of simplicity, the domains of initial conditions and controls are all extended to $[-h, 0) \cup \mathbf{R}_{0+}$ by zeroing them on the irrelevant intervals of $[-h, 0)$ so that any solution for $t \in \mathbf{R}_{0+}$ of (2.1) is identical to the corresponding one under the above given definition domains of vector functions of initial conditions and controls.

Theorem 2.1. The linear and time- invariant differential functional fractional dynamic system (2.1) of any order $\alpha \in \mathbf{C}_{0+}$ has a unique continuous solution on $[-h, 0] \cup \mathbf{R}_{0+}$ satisfying

(a) $x \equiv \varphi \equiv \sum_{j=0}^{k-1} \varphi_{j}$ on $\mathbf{R}_{0+}$ with $\varphi_{j}(0)=x_{j}(0)=x^{(j)}(0)=x_{j 0} ; j \in \overline{k-1} \cup\{0\}$; for all $t \in$ $[-h, 0)$ for each given set of initial functions and $\varphi_{j}:[-h, 0] \rightarrow \mathbf{R}^{n}, j \in \overline{k-1} \cup\{0\}$ being bounded piecewise continuous with eventual discontinuities in a set of zero measure of $[-h, 0] \subset \mathbf{R}$ of bounded discontinuities, that is, $\left.\varphi_{j} \in \mathrm{BPC}^{(0)}([-h, 0]), \mathbf{R}^{n}\right) ; j \in \overline{k-1} \cup\{0\}$ and each given bounded piecewise continuous control $u: \mathbf{R}_{0_{+}} \rightarrow \mathbf{R}^{m}$, with $u(t)=0$ for $t \in[-h, 0)$, being a bounded piecewise continuous control function, and

(b)

$$
\begin{aligned}
x_{\alpha}(t)= & \sum_{j=0}^{k-1}\left(\Phi_{\alpha j}(t) x_{j 0}+\sum_{i=1}^{r} \int_{0}^{r_{i}} \Phi_{\alpha}(t-\tau) A_{i} \varphi_{j}\left(\tau-r_{i}\right) d \tau\right. \\
& \left.\quad+\sum_{i=1}^{r} \int_{0}^{r_{i}} \Phi_{\alpha}(t-\tau) \tilde{A}_{i}(\tau) \varphi_{j}\left(\tau-r_{i}\right) d \tau\right) \\
& +\sum_{i=1}^{r} \int_{r_{i}}^{t} \Phi_{\alpha}(t-\tau) A_{i}(\tau) x_{\alpha}\left(\tau-r_{i}\right) d \tau+\sum_{i=0}^{r} \int_{r_{i}}^{t} \Phi_{\alpha}(t-\tau) \tilde{A}_{i}(\tau) x_{\alpha}\left(\tau-r_{i}\right) d \tau \\
& +\int_{0}^{t} \Phi_{\alpha}(t-\tau) B(\tau) u(\tau) d \tau, \quad t \in \mathbf{R}_{0+}
\end{aligned}
$$

which is time-differentiable satisfying (2.1) in $\mathbf{R}_{+}$with $k=[\operatorname{Re} \alpha]+1$ if $\alpha \notin \mathbf{Z}_{+}$and $k=\alpha$ if $\alpha \in \mathbf{Z}_{+}$, and

$$
\begin{gathered}
\Phi_{\alpha j}(t):=t^{j} E_{\alpha, j+1}\left(A_{0} t^{\alpha}\right), \quad \Phi_{\alpha}(t):=t^{\alpha-1} E_{\alpha \alpha}\left(A_{0} t^{\alpha}\right), \\
E_{\alpha j}\left(A_{0} t^{\alpha}\right):=\sum_{\ell=0}^{\infty} \frac{\left(A_{0} t^{\alpha}\right)^{\ell}}{\Gamma(\alpha \ell+j)}, \quad j \in \overline{k-1} \cup\{0, \alpha\},
\end{gathered}
$$

for $t \in \mathbf{R}_{0+}$ and $\Phi_{\alpha 0}(t)=\Phi_{\alpha}(t)=0$ for $t<0$, where $E_{\alpha, j}\left(A_{0} t^{\alpha}\right)$ are the Mittag-Leffler functions. 
A technical result about norm upper-bounding functions of the matrix functions (2.3)(2.4) follows

Lemma 2.2. The following properties hold.

(i) There exist finite real constants $K_{E \alpha j} \geq 1, K_{\Phi \alpha j} \geq 1 ; j \in \overline{k-1} \cup\{0\}$ and $K_{\Phi \alpha} \geq 1$ such that for any $\alpha\left(\in \mathbf{R}_{+}\right)<1$

$$
\begin{aligned}
E_{\alpha j}\left(A_{0} t^{\alpha}\right) & \leq K_{E \alpha j}\left\|e^{A_{0} t}\right\|, \quad\left\|\Phi_{\alpha j}(t)\right\| \leq K_{\Phi \alpha j}\left\|t^{j} e^{A_{0} t}\right\|, \quad j \in \overline{k-1} \cup\{0, \alpha\}, \\
\left\|\Phi_{\alpha}(t)\right\| & \leq K_{\Phi \alpha}\left\|\left(1 / t^{1-\alpha}\right) e^{A_{0} t}\right\|, \quad \text { for } t\left(\in \mathbf{R}_{+}\right) \geq 1 .
\end{aligned}
$$

(ii) If $\alpha\left(\in \mathbf{R}_{+}\right) \geq 1$ then

$$
\begin{aligned}
\left\|E_{\alpha j}\left(A_{0} t^{\alpha}\right)\right\| & =\sum_{\ell=0}^{\infty}\left(\frac{\ell !}{\Gamma(\alpha \ell+j)}\right)\left(\frac{A_{0}^{\ell} t^{\alpha \ell}}{\ell !}\right) \leq \sup _{\ell \in \mathbf{Z}_{0+}}\left(\frac{\ell !}{\Gamma(\ell+1)}\right)\left\|e^{A_{0}\left(t^{\alpha}\right)}\right\| \\
& =\left\|e^{A_{0}\left(t^{\alpha}\right)}\right\|, \quad j \in \overline{k-1} \cup\{0\}, t \in \mathbf{R}_{0+}, \\
\left\|\Phi_{\alpha j}(t)\right\| & \leq \sup _{\ell \in \mathbf{Z}_{0+}}\left(\frac{\ell !}{\Gamma(\alpha \ell+j+1)}\right) t^{j}\left\|e^{A_{0}\left(t^{\alpha}\right)}\right\| \leq t^{j}\left\|e^{A_{0}\left(t^{\alpha}\right)}\right\|, \quad j \in \overline{k-1} \cup\{0\}, t \in \mathbf{R}_{0+}, \\
\left\|\Phi_{\alpha}(t)\right\| & \leq \sup _{\ell \in \mathbf{Z}_{0+}}\left(\frac{\ell !}{\Gamma((\ell+1) \alpha)}\right) t^{\alpha-1}\left\|e^{A_{0}\left(t^{\alpha}\right)}\right\| \leq t^{\alpha-1}\left\|e^{A_{0}\left(t^{\alpha}\right)}\right\|, \quad t \in \mathbf{R}_{0+} .
\end{aligned}
$$

If, in addition, $A_{0}$ is a stability matrix then $\left\|e^{A_{0} t}\right\| \leq K e^{-\lambda t}$ and $\left\|e^{A_{0}\left(t^{\alpha}\right)}\right\| \leq K e^{-\lambda t^{\alpha}} \leq K e^{-\lambda t} ; t \in \mathbf{R}_{0+}$ for some real constants $K \geq 1, \lambda \in \mathbf{R}_{+}$. Then, one gets from (2.5)

$$
\begin{gathered}
\left\|E_{\alpha j}\left(A_{0} t^{\alpha}\right)\right\| \leq K e^{-\lambda t}, \quad\left\|\Phi_{\alpha j}(t)\right\| \leq t^{j} e^{-\lambda t}, \quad j \in \overline{k-1} \cup\{0\}, \\
\left\|\Phi_{\alpha}(t)\right\| \leq t^{\alpha-1} e^{-\lambda t}
\end{gathered}
$$

for $t \in \mathbf{R}_{0+}$, and the fractional dynamic system in the absence of delayed dynamics is exponentially stable if the standard fractional system for $\alpha=1$ is exponentially stable.

(iii) The following inequalities hold.

$$
\begin{gathered}
\left\|\Phi_{\alpha, k-1}(t)\right\| \leq t^{k-\alpha}\left\|\Phi_{\alpha}(t)\right\| \quad \text { for } \alpha \in(k-1, k] \cap \mathbf{R}_{+} \text {for } k \in \mathbf{Z}_{+}, t \in \mathbf{R}_{0+}, \\
\left\|\Phi_{\alpha}(t)\right\| \leq t^{\alpha+1-k}\left\|\Phi_{\alpha, k-2}(t)\right\| \quad \text { for } \alpha \in[k-1, k) \cap \mathbf{R}_{+}, t \in \mathbf{R}_{0+}, \\
\left\|\Phi_{k}(t)\right\| \equiv\left\|\Phi_{k, k-1}(t)\right\| \quad \text { for } \alpha=k \in \mathbf{Z}_{+}, t \in \mathbf{R}_{0+} .
\end{gathered}
$$


Proof. Note from (2.3)-(2.4) for $0<\alpha\left(\in \mathbf{R}_{+}\right)<1$

$$
\begin{aligned}
& E_{\alpha j}\left(A_{0} t^{\alpha}\right):=\sum_{\ell=0}^{\infty} \frac{\left(A_{0} t^{\alpha}\right)^{\ell}}{\Gamma(\alpha \ell+j)} \\
& =\sum_{\ell=0}^{\infty}\left(\frac{\ell !}{\Gamma(\alpha \ell+j)}\right)\left(\frac{A_{0}^{\ell} t^{\alpha \ell}}{\ell !}\right)=\sum_{\ell=0}^{\infty}\left(\frac{t^{(\alpha-1) \ell} \ell !}{\Gamma(\alpha \ell+j)}\right)\left(\frac{A_{0}^{\ell} t^{\ell}}{\ell !}\right) \\
& \left\|E_{\alpha j}\left(A_{0} t^{\alpha}\right)\right\| \leq \sup _{\tau \in(1, \infty) \cap \mathbf{R}}\left(\sup _{\ell \in \mathbf{Z}_{0+}}\left(\frac{\ell !}{\tau^{(1-\alpha) \ell} \Gamma(\alpha \ell+j)}\right)\right)\left\|e^{A_{0} t}\right\|, \quad j \in \overline{k-1} \cup\{0, \alpha\}, \\
& t \in(1, \infty) \cap \mathbf{R}_{+}, \\
& \limsup _{t \rightarrow \infty}\left\|E_{\alpha, j}\left(A_{0} t^{\alpha}\right)\right\| \leq \limsup _{t \rightarrow \infty}\left(\sup _{\ell \in \mathbf{Z}_{0+}}\left(\frac{\ell !}{t^{(1-\alpha) \ell} \Gamma(\alpha \ell+j)}\right)\left\|\sum_{\ell=0}^{\infty} \frac{A_{0}^{\ell} t^{\ell}}{\ell !}\right\|\right) \\
& \leq \limsup _{t \rightarrow \infty}\left\|e^{A_{0} t}\right\|, \quad j \in \overline{k-1} \cup\{0, \alpha\} \text {, } \\
& \left\|\Phi_{\alpha j}(t)\right\| \leq \sup _{\tau \in(1, \infty) \cap \mathbf{R}}\left(\sup _{\ell \in \mathbf{Z}_{0+}}\left(\frac{\ell !}{\tau^{(1-\alpha) \ell} \Gamma(\alpha \ell+j+1)}\right)\right)\left\|t^{j} e^{A_{0} t}\right\|, \quad j \in \overline{k-1} \cup\{0, \alpha\}, \\
& t \in(1, \infty) \cap \mathbf{R}_{+}, \\
& \limsup _{t \rightarrow \infty}\left\|\Phi_{\alpha j}(t)\right\| \leq \limsup _{t \rightarrow \infty}\left(\sup _{\ell \in \mathbf{Z}_{0+}}\left(\frac{\ell !}{t^{(1-\alpha) \ell} \Gamma(\alpha \ell+j+1)}\right)\left\|\sum_{\ell=0}^{\infty} \frac{A_{0}^{\ell} t^{\ell+j}}{\ell !}\right\|\right) \\
& \leq \limsup _{t \rightarrow \infty}\left\|t^{j} e^{A_{0} t}\right\|, \quad j \in \overline{k-1} \cup\{0\}, \\
& \left\|\Phi_{\alpha}(t)\right\| \leq \sup _{\tau \in(1, \infty) \cap \mathbf{R}}\left(\sup _{\ell \in \mathbf{Z}_{0+}}\left(\frac{\ell !}{\tau^{(1-\alpha) \ell} \Gamma((\ell+1) \alpha)}\right)\right)\left\|\frac{1}{t^{1-\alpha}} e^{A_{0} t}\right\|, \quad t \in(1, \infty) \cap \mathbf{R}_{+}, \\
& \limsup _{t \rightarrow \infty}\left\|\Phi_{\alpha}(t)\right\| \leq \limsup _{t \rightarrow \infty}\left(\sup _{\ell \in \mathbf{Z}_{0+}}\left(\frac{\ell !}{t^{(1-\alpha) \ell} \Gamma((\ell+1) \alpha)}\right)\left\|\sum_{\ell=0}^{\infty} \frac{A_{0}^{\ell} t^{\ell+\alpha-1}}{\ell !}\right\|\right) \\
& \leq \limsup _{t \rightarrow \infty}\left\|\frac{1}{t^{1-\alpha}} e^{A_{0} t}\right\|
\end{aligned}
$$

since

$$
\limsup _{[\ell, \ell+1) \ni t\left(\in \mathbf{R}_{0+}\right) \rightarrow \infty}\left(\sup _{\ell \in \mathbf{Z}_{0+}}\left(\frac{\ell !}{t^{(1-\alpha) \ell} \Gamma((\ell+1) \alpha)}\right)\right) \leq \limsup _{\mathbf{Z}_{0+} \ni \ell \rightarrow \infty}\left(\sup _{\ell \in \mathbf{Z}_{0+}}\left(\frac{\ell !}{\ell^{(1-\alpha) \ell} \Gamma(\alpha \ell+j+1)}\right)\right)=0 .
$$


The inequalities (2.4) hold since the above matrix norms are bounded on the real interval $(1, \infty)$ and their limit superior is upper-bounded by the given formulas and Property (i) is proved. On the other hand, if $\mathbf{R}_{+} \ni \alpha \geq 1$ then

$$
\begin{aligned}
\left\|E_{\alpha j}\left(A_{0} t^{\alpha}\right)\right\| & =\sum_{\ell=0}^{\infty}\left(\frac{\ell !}{\Gamma(\alpha \ell+j)}\right)\left(\frac{A_{0}^{\ell} t^{\alpha \ell}}{\ell !}\right) \\
& \leq \sup _{\ell \in \mathbf{Z}_{0+}}\left(\frac{\ell !}{\Gamma(\ell+1)}\right)\left\|e^{A_{0}\left(t^{\alpha}\right)}\right\|=\left\|e^{A_{0}\left(t^{\alpha}\right)}\right\|, \quad j \in \overline{k-1} \cup\{0\}, t \in \mathbf{R}_{0+} \\
\left\|\Phi_{\alpha j}(t)\right\| & \leq \sup _{\ell \in \mathbf{Z}_{0+}}\left(\frac{\ell !}{\Gamma(\alpha \ell+j+1)}\right) t^{j}\left\|e^{A_{0}\left(t^{\alpha}\right)}\right\| \leq t^{j}\left\|e^{A_{0}\left(t^{\alpha}\right)}\right\|, \quad j \in \overline{k-1} \cup\{0\}, t \in \mathbf{R}_{0+}, \\
\left\|\Phi_{\alpha}(t)\right\| & \leq \sup _{\ell \in \mathbf{Z}_{0_{+}}}\left(\frac{\ell !}{\Gamma((\ell+1) \alpha)}\right) t^{\alpha-1}\left\|e^{A_{0}\left(t^{\alpha}\right)}\right\| \leq t^{\alpha-1}\left\|e^{A_{0}\left(t^{\alpha}\right)}\right\|, \quad t \in \mathbf{R}_{0+} .
\end{aligned}
$$

If, in addition, $A_{0}$ is a stability matrix then $\left\|e^{A_{0}\left(t^{\alpha}\right)}\right\| \leq K e^{-\lambda t}$ and $\left\|e^{A_{0}\left(t^{\alpha}\right)}\right\| \leq K e^{-\lambda t^{\alpha}} \leq K e^{-\lambda t}, t \in$ $\mathbf{R}_{0+}$ for some real constants $K \geq 1$ and $\lambda \in \mathbf{R}_{+}$since $t^{\alpha} \geq t$, for all $t \in \mathbf{R}_{0+}$. Properties (i)-(ii) have been proved.

(iii) It is proved as follows. Note from (2.3)-(2.4) that

$$
\left\|\Phi_{\alpha j}(t)\right\|=\left\|\sum_{\ell=0}^{\infty} \frac{A_{0}^{\ell} t^{\alpha \ell+j}}{\Gamma(\alpha \ell+j+1)}\right\| \leq \sup _{\ell \in \mathbf{Z}_{0+}}\left(\frac{\Gamma((\ell+1) \alpha) t^{1+j-\alpha}}{\Gamma(\alpha \ell+j+1)}\right)\left\|\sum_{\ell=0}^{\infty} \frac{A_{0}^{\ell} t^{(\ell+1) \alpha-1}}{\Gamma((\ell+1) \alpha)}\right\|, \quad t \in \mathbf{R}_{0+},
$$

$j \in \overline{k-1} \cup\{0\}$, so that if $k-1<\alpha\left(\in \mathbf{R}_{+}\right) \leq k$, then

$$
\left\|\Phi_{\alpha, k-1}(t)\right\| \leq \sup _{\ell \in \mathbf{Z}_{0+}}\left(\frac{\Gamma((\ell+1) \alpha) t^{1+j-\alpha}}{\Gamma(\alpha \ell+k)}\right)\left\|\sum_{\ell=0}^{\infty} \frac{A_{0}^{\ell} t^{(\ell+1) \alpha-1}}{\Gamma((\ell+1) \alpha)}\right\| \leq t^{k-\alpha}\left\|\Phi_{\alpha}(t)\right\|, \quad t \in \mathbf{R}_{0+}
$$

Also,

$$
\begin{aligned}
\left\|\Phi_{\alpha}(t)\right\| & =\left\|\sum_{\ell=0}^{\infty} \frac{A_{0}^{\ell} t^{(\ell+1) \alpha-1}}{\Gamma((\ell+1) \alpha)}\right\| \leq t^{\alpha-j-1} \sup _{\ell \in \mathbf{Z}_{0+}}\left(\frac{\Gamma(\alpha \ell+j+1)}{\Gamma((\ell+1) \alpha)}\right)\left\|\sum_{\ell=0}^{\infty} \frac{A_{0}^{\ell} t^{\alpha \ell+j}}{\Gamma(\alpha \ell+j+1)}\right\| \\
& \leq t^{\alpha-j-1}\left\|\Phi_{\alpha j}(t)\right\|, \quad t \in \mathbf{R}_{0+},
\end{aligned}
$$

if $\sup _{\ell \in \mathbf{Z}_{0+}}(\Gamma(\alpha \ell+j+1) / \Gamma((\ell+1) \alpha))<\infty$. This implies that

$$
\begin{gathered}
\left\|\Phi_{\alpha}(t)\right\| \leq t^{\alpha+1-k}\left\|\Phi_{\alpha, k-2}(t)\right\| \quad \text { for } \alpha \in[k-1, k) \cap \mathbf{R}_{+}, \\
\left\|\Phi_{k}(t)\right\| \equiv\left\|\Phi_{k, k-1}(t)\right\| \quad \text { for } \alpha \in[k-1, k) \cap \mathbf{R}_{+} .
\end{gathered}
$$




\section{Fixed Point Results}

A technical definition is now given to facilitate the subsequent result about fixed point. Property (ii) has been proven.

Definition 3.1. $S(\bar{\varphi}, u)$ is the set of all the piecewise continuous $n$-vector function from $[-h, 0) \cup \mathbf{R}_{0+}$ to $\mathbf{R}^{n}$ being time- differentiable in $\mathbf{R}_{+}$which are solutions of (2.1) for all admissible $k$-tuples of initial conditions $\bar{\varphi}:=\left(\varphi_{0}, \varphi_{1}, \ldots, \varphi_{k-1}\right)$ with $\left.\varphi_{j} \in \operatorname{BPC}^{(0)}([-h, 0]), \mathbf{R}^{n}\right)$ and controls $u \in \mathrm{BPC}^{(0)}\left(\mathbf{R}_{0+}, \mathbf{R}^{n}\right)$ with $\varphi_{j}(0)=x_{j}(0)=x^{(j)}(0)=x_{j 0}$; for all $j \in \overline{k-1} \cup\{0\}$.

A fixed point theorem is now given for the Caputo fractional system (2.1).

Theorem 3.2. Assume any set of $r$ given finite delays $0=r_{0}<r_{1} \leq \ldots \leq r_{r}=h<\infty$. The following properties hold. be defined by

(i) Assume that $\Phi_{\alpha j} \in L_{\infty}\left(\mathbf{R}_{0+}, \mathbf{R}^{n \times n}\right)$ and $\int_{0}^{\delta}\left\|\Phi_{\alpha}(\delta-\tau) d \tau\right\|\left\|\tilde{A}_{0}\right\|_{\infty}<1$; let $g_{h}: \mathbf{R}_{+} \rightarrow \mathbf{R}_{0+}$

$$
\begin{aligned}
g_{h}(\delta):= & \left(1-\int_{0}^{\delta}\left\|\Phi_{\alpha}(\delta-\tau) d \tau\right\|\left\|\tilde{A}_{0}\right\|_{\infty}\right)^{-1} \\
& \times\left(\left\|\sum_{j=0}^{k-1} \Phi_{\alpha j}(\delta)\right\|+\left(\left\|\int_{0}^{\delta} \Phi_{\alpha}(\delta-\tau) d \tau\right\|\right)\left(\sum_{i=1}^{r}\left\|\widehat{A}_{i}\right\|_{\infty}\right)\right) \leq 1, \quad \delta \in \mathbf{R}_{+} .
\end{aligned}
$$

Then, the mapping $f_{h}:[-h, 0] \times \mathbf{R}^{n} \rightarrow \mathbf{R}_{+} \times \mathbf{R}^{n}$ defined by the state trajectory solution (2.2) of the uncontrolled system from any initial conditions in the admissible set is nonexpansive, and the solution is bounded fulfilling $\sup _{t \in \mathbf{R}_{0_{+}}}\left\|x_{\alpha}(t)\right\|_{\infty} \leq \sup _{t \in[-h, 0]} \sum_{j=0}^{k-1}\left(\left\|\varphi_{j}(t)\right\|_{\infty}\right)$. If $g_{h}(\delta) \leq K_{c}(\delta)<$ 1; for all $\in \mathbf{R}_{+}$then $f_{h}:[-h, 0] \times \mathbf{R}^{n} \rightarrow \mathbf{R}_{+} \times \mathbf{R}^{n}$ is contractive and possesses a unique fixed point, irrespective of the delays, in some bounded subset of $\mathbf{R}^{n}$. Such a fixed point is $0 \in \mathbf{R}^{n}$ which is also a globally asymptotically stable equilibrium point.

(ii) Assume that $\Phi_{\alpha j} \in L_{\infty}\left(\mathbf{R}_{0+}, \mathbf{R}^{n \times n}\right), \Phi_{\alpha} \in L^{2}\left(\mathbf{R}_{0+}, \mathbf{R}^{n \times n}\right)$ and $\int_{0}^{\mathcal{\delta}}\left\|\Phi_{\alpha}(\delta-\tau) d \tau\right\|\left(\int_{0}^{\mathcal{\delta}} \| \tilde{A}_{0}(t+\right.$ $\left.\tau) \|^{2} d \tau\right)^{1 / 2}<1$; for all $t \in \mathbf{R}_{0+}$ and define pointwise $\widehat{g}_{h}: \mathbf{R}_{0+} \times \mathbf{R}_{+} \rightarrow \mathbf{R}_{0+}$ as follows

$$
\begin{aligned}
\widehat{g}_{h}(t, \delta):= & \left(1-\int_{0}^{\delta}\left\|\Phi_{\alpha}(\delta-\tau) d \tau\right\|\left(\int_{0}^{\delta}\left\|\tilde{A}_{0}(t+\tau)\right\|^{2} d \tau\right)^{1 / 2}\right)^{-1} \\
& \times\left(\sum_{j=0}^{k-1}\left\|\Phi_{\alpha j}(\delta)\right\|_{\infty}+\left(\int_{0}^{\delta}\left\|\Phi_{\alpha}(\delta-\tau)\right\|^{2} d \tau\right)^{1 / 2}\right. \\
& \left.\times\left(\sum_{i=1}^{r}\left(\int_{0}^{\delta}\left\|\widehat{A}_{i}\left(t+\tau-r_{i}\right)\right\|^{2}\right)^{1 / 2} d \tau\right)\right), \quad \delta \in \mathbf{R}_{+} .
\end{aligned}
$$

Then, Property (i) still holds by replacing their corresponding constraints on $g_{h}$ by corresponding ones on $\widehat{g}_{h}$.

(iii) Assume that a control $u(t)=\sum_{i=0}^{r} K_{i}\left(x_{t}, t\right) x\left(t-r_{i}\right)$ is injected to (2.1) where $K_{i}$ : $\mathbf{R}^{n} \times \mathbf{R}_{0+} \rightarrow \mathbf{R}^{m}$ is in $\operatorname{BPC}\left(\mathbf{R}_{0+}, \mathbf{R}^{m}\right), x_{i t}:\left[\max \left(0, t-r_{i}\right), t\right] \rightarrow \mathbf{R}^{\mathbf{n}}$, for all $i \in \overline{r-1} \cup\{0\}$, for all 
$t \in \mathbf{R}_{0+}$ is a strip of the state-trajectory solution of (2.1). Assume also that

$$
\begin{array}{r}
\left\|K_{i}\left(x_{i t}, t\right)\right\|_{\infty} \leq K_{i}^{0}<\infty, \quad \forall i \in \overline{r-1} \cup\{0\}, \forall t \in \mathbf{R}_{0+}, \Phi_{\alpha j} \in L_{\infty}\left(\mathbf{R}_{0+}, \mathbf{R}^{n \times n}\right), \\
\Phi_{\alpha} \in L^{1}\left(\mathbf{R}_{0+}, \mathbf{R}^{n \times n}\right),
\end{array}
$$

and define $\widehat{g}_{f}: \mathbf{R}_{+} \rightarrow \mathbf{R}_{0+}$ as

$$
\begin{aligned}
g_{h}(\delta):= & \left(1-\int_{0}^{\delta}\left\|\Phi_{\alpha}(\delta-\tau) d \tau\right\|\left(\left\|\tilde{A}_{0}\right\|_{\infty}+\|B\|_{\infty} K_{0}^{0}\right)\right)^{-1} \\
& \times\left(\left\|\sum_{j=0}^{k-1} \Phi_{\alpha j}(\delta)\right\|+\left(\left\|\int_{0}^{\delta} \Phi_{\alpha}(\delta-\tau) d \tau\right\|\right)\left(\sum_{i=1}^{r}\left(\left\|\widehat{A}_{i}\right\|_{\infty}+\|B\|_{\infty} K_{i}^{0}\right)\right)\right) \leq 1,
\end{aligned}
$$

$\delta \in \mathbf{R}_{+}$,

provided that $\int_{0}^{\delta}\left\|\Phi_{\alpha}(\delta-\tau) d \tau\right\|\left(\left\|\tilde{A}_{0}\right\|_{\infty}+\|B\|_{\infty} K_{0}^{0}\right)<1$. Then, for any given set of finite delays, the mapping $f_{f}:[-h, 0] \times \mathbf{R}^{n} \times \mathbf{R}^{m} \times \mathbf{R}_{0+} \rightarrow \mathbf{R}_{+} \times \mathbf{R}^{n}$ defined by the state trajectory solution (2.2) of the controlled system from any initial conditions in the admissible set and any given admissible control is a nonexpansive mapping if $g_{h}(\delta) \leq 1$; for all $\delta \in \mathbf{R}_{+}$and contractive and the zero equilibrium is the unique fixed point, irrespective of the delays and control, if $g_{h}(\delta) \leq K_{c}(\delta)<1$; for all $\delta \in \mathbf{R}_{+}$which is also a globally asymptotically stable equilibrium point.

(iv) Assume that $\exists \varepsilon(<1) \in \mathbf{R}_{+}$that $g_{h}(\delta)<1-\varepsilon$; for all $t \in \mathbf{R}_{0_{+}}$. Then, state trajectory solution (2.2) of the forced system from any initial conditions in the admissible set is defined by a contractive self-mapping with a unique fixed point in some bounded subset of $\mathbf{R}^{n}$ for all controls of the form $u(t)=\sum_{i=0}^{r} K_{i}\left(x_{i t}, t\right) x\left(t-r_{i}\right)$ fulfilling $\left\|K_{i}\left(x_{i t}, t\right)\right\|_{\infty} \leq \varepsilon /(r+1)\left(\left\|\int_{0}^{\delta} \Phi_{\alpha}(\delta-\tau) d \tau\right\|\right)\|B\|_{\infty}$, for all $i \in \overline{r-1} \cup\{0\}$.

(v) Assume that $\Phi_{\alpha j} \in L_{\infty}\left(\mathbf{R}_{0+}, \mathbf{R}^{n \times n}\right)$; for all $j \in \overline{k-1} \cup\{0\}, \Phi_{\alpha} \in L^{2}\left(\mathbf{R}_{0+}, \mathbf{R}^{n \times n}\right)$ and $B K_{i} \in$ $L^{2}\left(\mathbf{R}_{0+}, \mathbf{R}^{n \times n}\right)$; for all $i \in \overline{r-1} \cup\{0\}$, instead of the hypotheses (3.3), and define $\widehat{g}_{f}: \mathbf{R}_{0+} \times \mathbf{R}_{+} \rightarrow \mathbf{R}_{0+}$ as:

$$
\begin{aligned}
\widehat{g}_{f}(t, \delta):= & \left(1-\int_{0}^{\delta}\left\|\Phi_{\alpha}(\delta-\tau) d \tau\right\|\right. \\
& \left.\quad \times\left(\left(\int_{0}^{\delta}\left\|\widetilde{A}_{0}(t+\tau)\right\|^{2} d \tau\right)^{1 / 2}+\left(\int_{0}^{\delta}\left\|B(t+\tau) K_{0}\left(x_{t+\tau}, t+\tau\right)\right\|^{2} d \tau\right)^{1 / 2}\right)\right)^{-1} \\
\times & \left(\sum_{j=0}^{k-1}\left\|\Phi_{\alpha j}(\delta)\right\|_{\infty}+\left(\int_{0}^{\delta}\left\|\Phi_{\alpha}(\delta-\tau)\right\|^{2} d \tau\right)^{1 / 2}\right. \\
\times & \left.\left(\sum_{i=1}^{r}\left(\int_{0}^{\delta}\left\|\widehat{A}_{i}\left(t+\tau-r_{i}\right)\right\|^{2}\right)^{1 / 2} d \tau+\left(\int_{0}^{\delta}\left\|B(t+\tau) K_{i}\left(x_{t+\tau}, t+\tau\right)\right\|^{2} d \tau\right)^{1 / 2}\right)\right)
\end{aligned}
$$


provided that the inverse exists on $\mathbf{R}_{0+}$. Then, Property (iii) still holds by replacing their corresponding constraints on $g_{f}$ by corresponding ones on $\widehat{g}_{f}$. If, in addition, $\exists \varepsilon(<1) \in \mathbf{R}_{+}, \delta=\delta(\varepsilon) \in \mathbf{R}_{+}$such that $\widehat{g}_{h}(\delta)<1-\varepsilon$; for all $t \in \mathbf{R}_{0+}$ then the mapping $f_{f}:[-h, 0] \times \mathbf{R}^{n} \times \mathbf{R}^{m} \times \mathbf{R}_{0_{+}} \rightarrow \mathbf{R}_{+} \times \mathbf{R}^{n}$ defining the state-trajectory solution from any set of admissible initial conditions and all controls $u(t)=\sum_{i=0}^{r} K_{i}\left(x_{i t}, t\right) x\left(t-r_{i}\right)$ being subject to

$$
\sum_{i=1}^{r} \int_{0}^{\delta}\left(\left\|B(t+\tau) K_{i}\left(x_{t+\tau}, t+\tau\right)\right\|^{2} d \tau\right)^{1 / 2} \leq \frac{\varepsilon}{\left(\int_{0}^{\delta}\left\|\Phi_{\alpha}(\delta-\tau)\right\|^{2} d \tau\right)^{1 / 2}\|B\|_{\infty}}, \quad \forall t \in \mathbf{R}_{+}
$$

is contractive with a unique fixed point, irrespective of the delays, which is $0 \in \mathbf{R}^{n}$ being a globally asymptotically stable equilibrium point.

Proof. The pointwise difference between two solutions $x(t)$ and $z(t)$ of (2.1) subject to respective piecewise continuous initial conditions $\varphi_{x}:[-h, 0] \rightarrow \mathbf{R}^{n}$ and $\varphi_{z}:[-h, 0] \rightarrow \mathbf{R}^{n}$ and respective controls $u_{x}, u_{y} \in \mathrm{BPC}^{(0)}\left(\mathbf{R}_{0+}, \mathbf{R}^{n}\right)$ is according to (2.2)

$$
\begin{aligned}
x_{\alpha}(t)-z_{\alpha}(t)= & \sum_{j=0}^{k-1}\left(\Phi_{\alpha j}(t)\left(x_{j 0}-z_{j 0}\right)+\sum_{i=1}^{r} \int_{0}^{r_{i}} \Phi_{\alpha}(t-\tau) A_{i}\left(\varphi_{x j}\left(\tau-r_{i}\right)-\varphi_{z j}\left(\tau-r_{i}\right)\right) d \tau\right) \\
& +\sum_{j=0}^{k-1} \sum_{i=1}^{r} \int_{0}^{r_{i}} \Phi_{\alpha}(t-\tau) \tilde{A}_{i}(\tau)\left(\varphi_{x j}\left(\tau-r_{i}\right)-\varphi_{z j}\left(\tau-r_{i}\right)\right) d \tau \\
& +\sum_{i=1}^{r} \int_{r_{i}}^{t} \Phi_{\alpha}(t-\tau) A_{i}\left(x_{\alpha}\left(\tau-r_{i}\right)-z_{\alpha}\left(\tau-r_{i}\right)\right) d \tau \\
& +\sum_{i=0}^{r} \int_{0}^{r_{i}} \Phi_{\alpha}(t-\tau) \tilde{A}_{i}(\tau)\left(x_{\alpha}\left(\tau-r_{i}\right)-z_{\alpha}\left(\tau-r_{i}\right)\right) d \tau \\
& +\int_{0}^{t} \Phi_{\alpha}(t-\tau) B(\tau)\left(u_{x}(\tau)-u_{z}(\tau)\right) d \tau, \quad t \in \mathbf{R}_{0+} .
\end{aligned}
$$

Note from (2.3) that $\Phi_{\alpha j}(0)=I_{n} / j$ !; for all $j \in \overline{k-1} \cup\{0\}$ what is used in the definition of the metric space $\left(M,\|\cdot\|_{\infty}\right)$ with the supremum metric $\|\cdot\|_{\infty}$

$$
\begin{gathered}
M:=\left\{\phi \in \operatorname{PBC}^{(0)}\left([-h, 0) \cup \mathbf{R}_{0+}, \mathbf{R}^{n}\right): \phi \in S(\bar{\phi}, u), \phi \equiv\left(\sum_{j=0}^{k-1} \phi_{j}\right) \in \operatorname{BPC}^{(0)}\left([-h, 0), \mathbf{R}^{n}\right),\right. \\
\left.\forall j \in \overline{k-1} \cup\{0\}, \phi_{u} \in M_{u}\right\},
\end{gathered}
$$


where

$$
M_{u}:=\left\{\phi \in \operatorname{PBC}^{(0)}\left(\mathbf{R}_{0+}, \mathbf{R}^{n}\right): \phi \in S(0, u), u \in \mathrm{BPC}^{(0)}\left(\mathbf{R}_{0+}, \mathbf{R}^{n}\right)\right\},
$$

where $\operatorname{BPC}^{(0)}\left(\mathbf{R}, \mathbf{R}^{n}\right)$ is the set of bounded continuous $n$-vector functions on $\mathbf{R}$. Now, define $P: M \rightarrow M$ as the subsequent piecewise bounded continuous function on $[-h, 0) \cup \mathbf{R}_{0+}$, which is bounded continuous on $\mathbf{R}_{+}$, that is, $\phi \in \mathrm{PBC}^{(0)}\left([-h, 0], \mathbf{R}^{n}\right), \phi \in \mathrm{BC}^{(0)}\left(\mathbf{R}_{+}, \mathbf{R}^{n}\right)$ and satisfies (2.1) on $\mathbf{R}_{+}$. One gets for any bounded piecewise continuous solution of (2.1)

$$
\begin{aligned}
(P(\phi, u))(t):= & \sum_{j=0}^{k-1} \Phi_{\alpha j}(t) \phi_{j}(0)+\int_{0}^{t} \Phi_{\alpha}(t-\tau) \tilde{A}_{0}(\tau) \phi(\tau) d \tau \\
& +\sum_{j=0}^{k-1} \sum_{i=1}^{r}\left(\int_{0}^{r_{i}} \Phi_{\alpha}(t-\tau) \widehat{A}_{i}(\tau) \phi_{j}\left(\tau-r_{i}\right) d \tau+\int_{r_{i}}^{t} \Phi_{\alpha}(t-\tau) \widehat{A}_{i}(\tau) \phi_{j}\left(\tau-r_{i}\right) d \tau\right) \\
& +\int_{0}^{t} \Phi_{\alpha}(t-\tau) B(\tau) u_{\phi}(\tau) d \tau
\end{aligned}
$$

Note that the supremum metric on $[-h, 0) \cup \mathbf{R}_{0+}$ is induced by the supremum norm on $[-h, 0) \cup$ $\mathbf{R}_{0+}$ so that it is then coincident with the supremum norm. Define the truncated $\phi_{a, b} \in M$ as $\phi_{a, b}(\tau)=\phi(\tau), \tau \in[a, b)$ and $\phi_{a, b}(\tau)=0, \tau \in[0, a) \cup[b, \infty) \subset \mathbf{R}_{0+} ;$ for all $a, b(>a) \in[a, b) \subseteq$ $\mathbf{R}_{0+}$, for all $t \in \mathbf{R}_{0+}$, for all $\phi \in M$ and note that $\|\phi\|_{a, b}:=\sup _{\tau \in[a, b)}\|\phi(\tau)\|=\left\|\phi_{a, b}\right\|_{\infty} \leq\|\phi\|_{\infty}$ and $\phi_{t} \in M$ is a simplified notation for the truncated $\phi \in M$ on $[0, \infty)$. Norms without subscripts mean, depending on context, vector or correspondingly induced matrix norms (as, for instance, the $\ell_{2}$-vector or induced matrix norms) or pointwise values of such norms for vector or matrix functions in the subsequent developments. Let $M_{t}$ be the space of truncated functions $\phi_{t} \in M$. Note that any truncated solution of (2.1) on any finite interval is always in $M$ so that one gets for any $\delta \in \mathbf{R}_{+}$from (3.10) in the most general controlled case with control $u(t)=\sum_{i=0}^{r} K_{i}\left(x_{t}, t\right) x\left(t-r_{i}\right)$

$$
\begin{aligned}
& \left\|\left(P\left(\phi, u_{\phi}\right)\right)(t+\delta)-\left(P\left(\eta, u_{\eta}\right)\right)(t+\delta)\right\| \\
& \quad \leq \sum_{j=0}^{k-1}\left\|\Phi_{\alpha j}(\delta)\right\|\left\|\phi_{j}-\eta_{j}\right\|_{t}+\left\|\int_{0}^{\delta} \Phi_{\alpha}(\delta-\tau) B(t+\tau) d \tau\right\|\left\|u_{\phi}-u_{\eta}\right\|_{t+\delta} \\
& \quad+\left(\sum_{i=1}^{r}\left\|\int_{0}^{\delta} \Phi_{\alpha}(\delta-\tau) \widehat{A}_{i}(t+\tau) d \tau\right\|_{t+\delta-r_{i}}+\int_{0}^{\delta}\left\|\Phi_{\alpha}(\delta-\tau) \tilde{A}_{0}(t+\tau) d \tau\right\|\right)\|\phi-\eta\|_{t+\delta}
\end{aligned}
$$




$$
\begin{aligned}
& \leq\left(\left\|\sum_{j=0}^{k-1} \Phi_{\alpha j}(\delta)\right\|+\sum_{i=1}^{r} \int_{0}^{\delta}\left\|\Phi_{\alpha}(\delta-\tau) B(t+\tau) K_{i}\left(x_{i, t+\tau}, t+\tau\right) d \tau\right\|\|\phi-\eta\|_{t+\delta-r_{i}}\right. \\
& \quad+\sum_{i=1}^{r} \int_{0}^{\delta}\left\|\Phi_{\alpha}(\delta-\tau) \widehat{A}_{i}(t+\tau) d \tau\right\|\|\phi-\eta\|_{t+\delta-r_{i}} \\
& \left.\quad+\int_{0}^{\delta}\left\|\Phi_{\alpha}(\delta-\tau)\left(\tilde{A}_{0}(t+\tau)+B(t+\tau) K_{0}(t+\tau)\right)\right\| d \tau\|\phi-\eta\|_{t+\delta}\right) \\
& \leq\left\|\sum_{j=0}^{k-1} \Phi_{\alpha j}(\delta)\right\|\|\phi-\eta\|_{t+\delta}+\sum_{i=1}^{r} \int_{0}^{\delta}\left\|\Phi_{\alpha}(\delta-\tau) d \tau\right\| \\
& \quad \times\left(\sum_{i=1}^{r}\left(\left\|\widehat{A}_{i}\right\| \infty+\|B\|_{\infty} K_{i}^{0}\right)\|\phi-\eta\|_{t+\delta-r_{i}}+\left(\left\|\tilde{A}_{0}\right\|_{\infty}+\|B\|_{\infty} K_{0}^{0}\right)\|\phi-\eta\|_{t+\delta}\right) \\
& \leq\left(\left\|\sum_{j=0}^{k-1} \Phi_{\alpha j}(\delta)\right\|+\sum_{i=1}^{r} \int_{0}^{\delta}\left\|\Phi_{\alpha}(\delta-\tau) d \tau\right\|\left(\sum_{i=1}^{r}\left(\left\|\widehat{A}_{i}\right\|_{\infty}+\|B\|_{\infty} K_{i}^{0}\right)+\left(\left\|\tilde{A}_{0}\right\|_{\infty}+\|B\|_{\infty} K_{0}^{0}\right)\right)\right) \\
& \quad \times\|\phi-\eta\|_{t+\delta},
\end{aligned}
$$

where the property that $A_{0}$ is constant has been used to rewrite the limits of the involved integral is the most convenient fashion to simplify the related expressions. Equation (3.13) leads to

$\|\phi-\eta\|_{t+\delta}$

$$
\begin{aligned}
& \leq\left(1-\int_{0}^{\delta}\left\|\Phi_{\alpha}(\delta-\tau) d \tau\right\|\left(\left\|\tilde{A}_{0}\right\|_{\infty}+\|B\|_{\infty}\left\|K_{0}^{0}\right\|_{\infty}\right)\right)^{-1} \\
& \times\left(\left\|\sum_{j=0}^{k-1} \Phi_{\alpha j}(\delta)\right\|+\sum_{i=1}^{r} \int_{0}^{\delta}\left\|\Phi_{\alpha}(\delta-\tau) d \tau\right\|\left(\sum_{i=1}^{r}\left(\left\|\widehat{A}_{i}\right\|_{\infty}+\|B\|_{\infty}\left\|K_{i}^{0}\right\|_{\infty}\right)\|\phi-\eta\|_{t+\delta-r_{i}}\right)\right) \\
& \leq\left(1-\int_{0}^{\delta}\left\|\Phi_{\alpha}(\delta-\tau) d \tau\right\|\left(\left\|\tilde{A}_{0}\right\|_{\infty}+\|B\|_{\infty}\left\|K_{0}^{0}\right\|_{\infty}\right)\right)^{-1} \\
& \times\left(\left\|\sum_{j=0}^{k-1} \Phi_{\alpha j}(\delta)\right\|+\sum_{i=1}^{r} \int_{0}^{\delta}\left\|\Phi_{\alpha}(\delta-\tau) d \tau\right\|\left(\sum_{i=1}^{r}\left(\left\|\widehat{A}_{i}\right\|_{\infty}+\|B\|_{\infty}\left\|K_{i}^{0}\right\|_{\infty}\right)\|\phi-\eta\|_{t+\delta-r_{1}}\right)\right), \\
& \forall \delta \in \mathbf{R}_{+}, \quad \forall t \in \mathbf{R}_{0+},
\end{aligned}
$$


provided that $\int_{0}^{\delta}\left\|\Phi_{\alpha}(\delta-\tau) d \tau\right\|\left(\left\|\widetilde{A}_{0}\right\|_{\infty}+\|B\|_{\infty} K_{0}^{0}\right)<1$, since $r_{1} \leq r_{i}(i \in \bar{r})$, so that

$$
\begin{aligned}
& \|\phi-\eta\|_{t} \\
& \leq\left(1-\int_{0}^{\delta}\left\|\Phi_{\alpha}(\delta-\tau) d \tau\right\|\left(\left\|\tilde{A}_{0}\right\|_{\infty}+\|B\|_{\infty} K_{0}^{0}\right)\right)^{-1} \\
& \quad \times \quad\left(\left\|\sum_{j=0}^{k-1} \Phi_{\alpha j}(\delta)\right\|+\sum_{i=1}^{r} \int_{0}^{\delta}\left\|\Phi_{\alpha}(\delta-\tau) d \tau\right\|\left(\sum_{i=1}^{r}\left(\left\|\widehat{A}_{i}\right\|_{\infty}+\|B\|_{\infty}\left\|K_{i}^{0}\right\|_{\infty}\right)\|\phi-\eta\|_{t-r_{1}}\right)\right) \\
& =g_{h}(\delta)\|\phi-\eta\|_{t-r_{1}} ; \quad \forall \delta \in \mathbf{R}_{+}, \forall t \in \mathbf{R}_{0+} .
\end{aligned}
$$

Then, the mapping $f_{h}:[-h, 0] \times \mathbf{R}^{n} \rightarrow \mathbf{R}_{+} \times \mathbf{R}^{n}$ defining the state trajectory solution from admissible initial conditions is nonexpansive if $g_{h}(\delta) \leq 1$. Furthermore, the state trajectory solution is globally Lyapunov stable since by taking the trivial solution $\eta \equiv 0$ in (3.15), it follows that any solution $\phi$ of (2.1) generated from any set of admissible initial conditions is uniformly bounded on $\mathbf{R}_{0+}$. If, in addition, $g_{h}(\delta) \leq K_{c}(\delta)<1$ then it follows also from (3.15) as $t \rightarrow \infty$ that any real sequence of the form $\left\{v\left(k r_{1}+\tau\right)\right\}_{k \in \mathbf{Z}_{0+}, \tau \in\left[0, r_{1}\right) \cap \mathbf{R}_{0_{+}}}$is a convergent Cauchy sequence to zero in the metric space $\left(M,\|\cdot\|_{\infty}\right)$ of the solutions of (2.1) under the class of given initial conditions and controls with the supremum metric $\|\cdot\|_{\infty}$ is complete. Therefore, a unique fixed point exists on some bounded set of $\mathbf{R}^{n}$ from Banach contraction principle. Since

$$
\lim _{\mathbf{z}_{0+} \ni k \rightarrow \infty, \tau \in\left[0, r_{1}\right) \cap \mathbf{R}_{0+}}\|\phi-\eta\|_{(k+1)\left(r_{1}+\tau\right)} \leq\left(\lim _{\mathbf{z}_{0+} \ni k \rightarrow \infty} K_{c}^{k}(\delta)\right)\|\phi-\eta\|_{k\left(r_{1}+\tau\right), \tau \in\left[0, r_{1}\right) \cap \mathbf{R}_{0+}}=0,
$$

it follows by taking one of the solutions to be the trivial solution that the only fixed point is the equilibrium point zero which is a globally asymptotically stable attractor. Property (i) has been proven. By zeroing the control and considering the uncontrolled system, one proves Property (i) as a particular case of Property (iii). Property (ii) (and its particular case Property (iv) for the case of controller gains satisfying $\left.\left\|K_{i}\left(x_{i t}, t\right)\right\|_{\infty} \leq K_{i}^{0}<\infty\right)$ and Property (v) are proved by using similar technical tools to those involved in the above proofs by replacing the basic inequality (3.13) by

$$
\begin{aligned}
\left\|\left(P\left(\phi, u_{\phi}\right)\right)(t)-\left(P\left(\eta, u_{\eta}\right)\right)(t)\right\| \\
\leq\left(\left\|\sum_{j=0}^{k-1} \Phi_{\alpha j}(t)\right\| \|_{t-r_{1}, t}+\sum_{i=1}^{r}\left(\int_{t-r_{i}}^{t}\left\|\Phi_{\alpha}(t-\tau)\right\|^{2} d \tau\right)^{1 / 2}\right. \\
\quad \times\left(\left(\int_{t-r_{i}}^{t}\left\|\widehat{A}_{i}(\tau)\right\|^{2}\right)^{1 / 2} d \tau+\int_{t-r_{i}}^{t}\left(\left\|B(\tau) K_{i}\left(x_{\tau}, \tau\right)\right\|^{2} d \tau\right)^{1 / 2}\right) \\
\left.\quad+\left(\int_{t-r_{1}}^{t}\left\|\tilde{A}_{0}(\tau)\right\|^{2} d \tau\right)^{1 / 2}\right)\|\phi-\eta\|_{t-r_{1},} \quad \forall \delta \in \mathbf{R}_{+}, \forall t \in \mathbf{R}_{0++} .
\end{aligned}
$$


If all the delays are zero, it is more convenient to discuss the adhoc solution version of (2.2):

$$
x_{\alpha}(t)=\sum_{j=0}^{k-1} \bar{\Phi}_{\alpha j}(t) x_{j 0}+\sum_{i=0}^{r} \int_{0}^{t} \bar{\Phi}_{\alpha}(t-\tau) \tilde{A}_{i}(\tau) x_{\alpha}(\tau) d \tau+\int_{0}^{t} \bar{\Phi}_{\alpha}(t-\tau) B(\tau) u(\tau) d \tau, \quad t \in \mathbf{R}_{0+}
$$

where $\bar{\Phi}_{\alpha j}(t)$ and $\bar{\Phi}_{\alpha}(t)$ are similar to $\Phi_{\alpha j}(t)$ from (2.3)-(2.4) by replacing $A_{0} \rightarrow\left(\sum_{i=0}^{r} A_{i}\right)$. The following result is a counterpart to Theorem 3.2 for the case of absence of delays.

Theorem 3.3. Assume that

(1) $\left\|\bar{\Phi}_{\alpha j}(t)\right\| \leq K_{0 j}(t) ; t \in \mathbf{R}_{0+}$, for all $j \in \overline{k-1} \cup\{0\}$ with $\max _{0 \leq j \leq k-1}\left(\sup _{t \in \mathbf{R}_{0+}} K_{0 j}(t)\right) \leq$ $\bar{K}_{0}<\infty$.

(2) $\bar{\Phi}_{\alpha} \in L^{1}\left(\mathbf{R}_{0+}, \mathbf{R}^{n \times n}\right)$ with $\sup _{t \in \mathbf{R}_{0+}}\left\|\bar{\Phi}_{\alpha}(t)\right\| \leq \bar{K}_{1}<\infty$.

Then, the Caputo delay-free fractional dynamic system (2.1) of real order a has the following properties.

(i) It is globally stable under a control $u(t)=\sum_{i=0}^{r} K_{i}\left(x_{i t}, t\right) x(t)$ subject to $\left\|K_{i}\left(x_{i t}, t\right)\right\|_{\infty} \leq$ $K_{i}^{0}<\infty$, for all $i \in \overline{r-1}\{0\}$ if $\bar{K}_{1}<\left(1 / \sum_{i=0}^{r}\left(\left\|\tilde{A}_{i}\right\|_{\infty}+\|B\|_{\infty} K_{i}^{0}\right)\right)$, for all $i \in \overline{r-1}\{0\}$. If, in addition, $K_{0 j}(t) \rightarrow 0$ as $t \rightarrow \infty$; for all $j \in \overline{k-1} \cup\{0\}$ then the system is globally asymptotically stable to the zero equilibrium point.

(ii) Property (i) holds if $K_{0}(t)=K_{0}^{0}$ is constant if $\bar{K}_{1}<1 / \sum_{i=0}^{r}\left\|\widetilde{A}_{i}\right\|_{\infty}+\|B\|_{\infty}\left(\sum_{i=1}^{r} K_{i}^{0}\right)$ where $\bar{\Phi}_{\alpha j}(t)$ and $\bar{\Phi}_{\alpha}(t)$ are similar to $\Phi_{\alpha j}(t)$ from (2.3)-(2.4) by replacing $A_{0} \rightarrow$ $\left(\sum_{i=0}^{r} A_{i}\right)+B K_{0}^{0}$.

Proof. (i) One gets, after taking norms in (3.18), that

$$
\begin{aligned}
\left\|x_{\alpha}(t)\right\| & \leq\left\|\sum_{j=0}^{k-1} \bar{\Phi}_{\alpha j}(t) x_{j 0}\right\|+\sum_{i=0}^{r}\left(\left\|\tilde{A}_{i}\right\|_{\infty}+\|B\| K_{i}^{0}\right)\left(\int_{0}^{t}\left\|\Phi_{\alpha}(t-\tau) d \tau\right\|\right) \sup _{\tau \in[0, t)}\left\|x_{\alpha}(\tau)\right\| \\
& \leq \sum_{j=0}^{k-1} K_{0 j}(t)\left\|x_{j 0}\right\|+\sum_{i=0}^{r}\left(\left\|\tilde{A}_{i}\right\|_{\infty}+\|B\| K_{i}^{0}\right)\left(\int_{0}^{t}\left\|\Phi_{\alpha}(t-\tau) d \tau\right\|\right) \sup _{\tau \in[0, t)}\left\|x_{\alpha}(\tau)\right\| \\
& \leq \sum_{j=0}^{k-1} K_{0 j}(t)\left\|x_{j 0}\right\|+\bar{K}_{1}\left(\sum_{i=0}^{r}\left(\left\|\tilde{A}_{i}\right\|_{\infty}+\|B\| K_{i}^{0}\right)\right) \sup _{\tau \in[0, t)}\left\|x_{\alpha}(\tau)\right\| \\
& \leq \bar{K}_{0}\left(\sum_{j=0}^{k-1}\left\|x_{j 0}\right\|\right)+\bar{K}_{1}\left(\sum_{i=0}^{r}\left(\left\|\tilde{A}_{i}\right\| \infty+\|B\| K_{i}^{0}\right)\right) \sup _{\tau \in[0, t)}\left\|x_{\alpha}(\tau)\right\|, \quad t \in \mathbf{R}_{0+},
\end{aligned}
$$


with $\sup _{\tau \in[0, t)}\left\|x_{\alpha}(\tau)\right\|=\left\|x_{\alpha}\right\|_{t} \leq\left\|x_{\alpha}\right\|_{\infty}$. Thus, one gets from (3.20)

$$
\begin{aligned}
\left\|x_{\alpha}(t)\right\| & \leq \sup _{\tau \in[0, t)}\left\|x_{\alpha}(\tau)\right\| \\
& \leq\left(1-\bar{K}_{1}\left(\sum_{i=0}^{r}\left(\left\|\tilde{A}_{i}\right\|_{\infty}+\|B\|_{\infty} K_{i}^{0}\right)\right)\right)^{-1} \bar{K}_{0}\left(\sum_{j=0}^{k-1}\left\|x_{j 0}\right\|\right) \leq \bar{K}_{2}<\infty, \quad t \in \mathbf{R}_{0+} .
\end{aligned}
$$

Since $\bar{K}_{1}\left(\sum_{i=0}^{r}\left(\left\|\tilde{A}_{i}\right\|_{\infty}+\|B\|_{\infty} K_{i}^{0}\right)\right)<1$, where $\bar{K}_{2}:=\sup _{t \in \mathbf{R}_{0_{+}}}\left\|x_{\alpha}(t)\right\|<\infty$. As a result, the Caputo fractional system of real order $\alpha$ is globally stable under zero delays since any state trajectory solution generated from any admissible initial conditions is bounded for all time. The proof of Property (ii) is similar to that of (i) under the modified constraints. Now, assume that if, in addition, $K_{0 j}(t) \rightarrow 0$ as $t \rightarrow \infty$; for all $j \in \overline{k-1} \cup\{0\}$, then

$$
\left\|x_{\alpha}(t)\right\| \leq \min \left(1,\left[\sum_{j=0}^{k-1} K_{0 j}(t)+\sum_{i=0}^{r}\left(\left\|\tilde{A}_{i}\right\|_{\infty}+\|B\|_{\infty} K_{i}^{0}\right) \bar{K}_{1}\right]\right) \bar{K}_{2}
$$

so that

$$
\begin{aligned}
\limsup _{t \rightarrow \infty}\left\|x_{\alpha}(t)\right\| & \leq \min \left(1, \limsup _{t \rightarrow \infty}\left[\sum_{j=0}^{k-1} K_{0 j}(t)+\sum_{i=0}^{r}\left(\left\|\tilde{A}_{i}\right\|_{\infty}+\|B\|_{\infty} K_{i}^{0}\right) \bar{K}_{1}\right]\right) \bar{K}_{2} \\
& =\left(\sum_{i=0}^{r}\left(\left\|\tilde{A}_{i}\right\|_{\infty}+\|B\|_{\infty} K_{i}^{0}\right) \bar{K}_{1}\right) \bar{K}_{2}<\bar{K}_{2} .
\end{aligned}
$$

Since $\lim _{t \rightarrow \infty} K_{0 j}(t)=0$; for all $j \in \overline{k-1} \cup\{0\}$ and $\sum_{i=0}^{r}\left(\left\|\widetilde{A}_{i}\right\|_{\infty}+\|B\| K_{i}^{0}\right) \bar{K}_{1}<1$. Equation (3.23) implies that the supremum $\left\|x_{\alpha}(t)\right\|$ on $\mathbf{R}_{0+}$ is reached by the first time at some finite time $t_{0} \in \mathbf{R}_{0+}$. Thus, one gets from (3.19) that

$$
\begin{aligned}
\lim _{t \rightarrow \infty}\left\|x_{\alpha}(t)\right\| & \leq \lim _{t \rightarrow \infty}\left(\sup _{\tau \in\left[t_{0}, t\right)}\left\|x_{\alpha}(\tau)\right\|\right) \\
& \leq\left(1-\bar{K}_{1}\left(\sum_{i=0}^{r}\left(\left\|\tilde{A}_{i}\right\|_{\infty}+\|B\|_{\infty} K_{i}^{0}\right)\right)\right)^{-1}\left(\sum_{j=0}^{k-1}\left\|x_{\alpha j t_{0}}\right\|\left(\lim _{t \rightarrow \infty}\left(K_{0 j}\left(t-t_{0}\right)\right)\right)\right) \\
& =0
\end{aligned}
$$

provided that $K_{0 j}(t) \rightarrow 0$ as $t \rightarrow \infty$; for all $j \in \overline{k-1} \cup\{0\}$ which proves the global asymptotic stability. Property (i) has been proven. Property (ii) follows in a similar way under the modified constraints $K_{0}(t)=K_{0}^{0} \bar{K}_{1}<\left(1 / \sum_{i=0}^{r}\left\|\widetilde{A}_{i}\right\|_{\infty}+\|B\|_{\infty}\left(\sum_{i=1}^{r} K_{i}^{0}\right)\right), \bar{\Phi}_{\alpha j}(t), \bar{\Phi}_{\alpha}(t)$ being similar to $\Phi_{\alpha j}(t)$ from (2.3)-(2.4) by replacing $A_{0} \rightarrow\left(\sum_{i=0}^{r} A_{i}\right)+B K_{0}^{0}$. 
The subsequent stability result is based on a transformation of the matrix $A_{0}$ to its diagonal Jordan form which allows an easy computation of the $\ell_{2}$-matrix measure of its diagonal part.

Theorem 3.4. Assume that $J_{A_{0}}=J_{A_{0 d}}+\widetilde{J}_{A_{0}}$ is the Jordan form of $A_{0}$ with $J_{A_{0 d}}$ being diagonal and $\tilde{J}_{A_{0}}$ being off-diagonal such that the above decomposition is unique with $A_{0}=T^{-1} J_{A_{0}} T$ where $T$ is a unique nonsingular transformation matrix. The following properties hold.

(i) The Caputo fractional differential system (2.1) is globally Lyapunov stable independent of the delays if the $\ell_{2}$-matrix measure of $J_{A_{0 d}}$ is negative, that is,

$$
\mu_{2}\left(J_{A_{0 d}}^{1 / \alpha}\right):=\frac{1}{2} \lambda_{\max }\left(J_{A_{0 d}}^{1 / \alpha}+J_{A_{0 d}}^{* 1 / \alpha}\right)=\max _{k \in \bar{n}} \operatorname{Re}\left(\lambda_{k}^{1 / \alpha}\right)<0, \quad \forall \lambda_{k} \in \sigma\left(J_{A_{0 d}}^{1 / \alpha}\right)
$$

where $\sigma\left(J_{A_{0 d}}^{1 / \alpha}\right)$ is the spectrum of $J_{A_{0 d}}^{1 / \alpha}$ and, furthermore,

$$
\left\|\left(\frac{1}{\beta_{0}} T^{-1} \widetilde{J}_{A_{0}} T, \frac{1}{\beta_{1}} T^{-1} A_{1} T, \frac{1}{\beta_{2}} T^{-1} A_{2} T, \ldots, \frac{1}{\beta_{r}} T^{-1} A_{r} T\right)\right\|_{2} \leq\left|\mu_{2}\left(J_{A_{0 d}}\right)\right|^{1 / \alpha}
$$

for some set of numbers $\beta_{i} \in \mathbf{R}_{+}(i \in \bar{p} \cup\{0\})$ satisfying $\sum_{i=0}^{r} \beta_{i}^{2}=1$. The fractional system is globally asymptotically Lyapunov stable for one such set of real numbers if $\mu_{2}\left(J_{A_{0 d}}^{1 / \alpha}\right)<0$, and

$$
\left\|\left(\frac{1}{\beta_{0}} T^{-1} \widetilde{J}_{A_{0}} T, \frac{1}{\beta_{1}} T^{-1} A_{1} T, \frac{1}{\beta_{2}} T^{-1} A_{2} T, \ldots, \frac{1}{\beta_{r}} T^{-1} A_{r} T\right)\right\|_{2}<\left|\mu_{2}\left(J_{A_{0 d}}\right)\right|^{1 / \alpha} .
$$

(ii) A necessary condition for $\mu_{2}\left(J_{A_{0 d}}^{1 / \alpha}\right)<0$ is that $A_{0}$ should be a stability matrix with $|\arg (\lambda)|<(\alpha \pi / 2)$; for all $\lambda \in \sigma\left(A_{0}\right)$. Such a condition holds directly if $\alpha>2 \varphi / \pi$ where $(-\varphi, \varphi) \subseteq(-\pi / 2, \pi / 2)$ is the symmetric maximum real interval containing the arguments of all $\lambda \in \sigma\left(A_{0}\right)$. It also holds, in particular, if $A_{0}$ is a stability matrix and $\alpha\left(\in \mathbf{R}_{+}\right) \geq 1$.

Proof. It follows by using the matrix similarity transformation $A_{0}=T^{-1} J_{A_{0}} T=T^{-1}\left(J_{A_{0 d}}+\widetilde{J}_{A_{0}}\right) T$ and using the homogeneous transformed Caputo fractional differential system from (2.1)

$$
\begin{aligned}
\left({ }^{C} D_{0+}^{\alpha} z\right)(t) & =\left({ }^{C} D_{0+}^{\alpha} T x\right)(t)=\sum_{i=0}^{r} A_{i} T x\left(t-h_{i}\right) \Longleftrightarrow \\
\left({ }^{C} D_{0+}^{\alpha} x\right)(t) & =\sum_{i=0}^{p} T^{-1} A_{i} T x\left(t-h_{i}\right)=T^{-1} A_{0} T x(t)+\sum_{i=1}^{r} T^{-1} A_{i} T x\left(t-h_{i}\right) \\
& =T^{-1} J_{A_{0 d}} T x(t)+\sum_{i=0}^{r} T^{-1} \bar{A}_{i} T x\left(t-h_{i}\right),
\end{aligned}
$$


where $z(t)=T x(t)$; for all $t \in \mathbf{R}_{0+}, h_{0}=0$ plays the role of an additional delay. $\bar{A}_{0}=\tilde{J}_{A_{0}}$ and $\bar{A}_{i}=A_{i}(i \in \bar{r})$ by noting also that since $\left(J_{A_{0 d}}+J_{A_{0 d}}^{*}\right)$ is diagonal with real eigenvalues by construction, one has

$$
\begin{aligned}
\left|\mu_{2}\left(J_{A_{0 d}}^{1 / \alpha}\right)\right| & =\left|\frac{1}{2} \lambda_{\max }\left(J_{A_{0 d}}^{1 / \alpha}+J_{A_{0 d}}^{1 / \alpha^{*}}\right)\right|=\left|\lambda_{\max }\left(J_{A_{0 d}}^{1 / \alpha}\right)\right| \\
& =\left|\operatorname{Re} \lambda_{\max }\left(J_{A_{0 d}}^{1 / \alpha}\right)\right|=\left|\operatorname{Re} \lambda_{\max }^{1 / \alpha}\left(J_{A_{0 d}}\right)\right|=\left|\operatorname{Re} \lambda_{\max }^{1 / \alpha}\left(A_{0 d}\right)\right| \\
& =\left|\mu_{2}\left(J_{A_{0 d}}\right)\right|^{1 / \alpha} .
\end{aligned}
$$

Then, the remaining part of the proof of Property (i) is similar to that quoted as a sufficient condition for stability independent of the delays in [27]. Property (i) has been proven.

To prove property (ii), note that

$$
\begin{aligned}
\lambda_{\max }\left(J_{A_{0 d}}^{1 / \alpha}+J_{A_{0 d}}^{* 1 / \alpha}\right) & \geq \frac{1}{2} \lambda_{\max }\left(J_{A_{0 d}}+J_{A_{0 d}}^{*}\right)^{1 / \alpha} \text { if } \mu_{2}\left(J_{A_{0 d}}^{1 / \alpha}\right)<0 \text { so that } \\
0>\mu_{2}\left(J_{A_{0 d}}^{1 / \alpha}\right) & =\frac{1}{2} \lambda_{\max }\left(J_{A_{0 d}}^{1 / \alpha}+J_{A_{0 d}}^{* 1 / \alpha}\right) \geq \frac{1}{2} \lambda_{\max }\left(J_{A_{0 d}}+J_{A_{0 d}}^{*}\right)^{1 / \alpha} \\
& =\max \left(\operatorname{Re} \hat{\jmath}: \hat{\jmath} \in \sigma\left(J_{A_{0 d}}^{1 / \alpha}\right)\right) \\
& \geq \frac{1}{2} \lambda_{\max }\left(J_{A_{0 d}}+J_{A_{0 d}}^{*}\right)^{1 / \alpha}=\max \left(\operatorname{Re} \lambda^{1 / \alpha}: \lambda \in \sigma\left(A_{0}\right) \equiv \sigma\left(J_{A_{0 d}}\right)\right) .
\end{aligned}
$$

Thus, $A_{0}$ is a stability matrix if and only if $\arg (\lambda) \in\left(-\theta_{1}, \theta_{2}\right) \subseteq(-\pi / 2, \pi / 2)$; for all $\lambda \in$ $\sigma\left(A_{0}\right)$. If also $J_{A_{0 d}}^{1 / \alpha}$ is a stability matrix with $\mu_{2}\left(J_{A_{0 d}}^{1 / \alpha}\right)<0$, then $(1 / \alpha) \arg (\lambda) \in\left(-\theta_{1} / \alpha, \theta_{2} / \alpha\right) \subseteq$ $(-\pi / 2, \pi / 2)$ so that $|\arg (\lambda)|<(\alpha \pi / 2)$; for all $\lambda \in \sigma\left(A_{0}\right)$, which is also a necessary condition for the fulfillment of the sufficiency-type condition (3.27) for global asymptotic stability of (2.1), which implies the stability of the matrix $J_{A_{0 d}}^{1 / \alpha}$ with the further constraint that $\mu_{2}\left(J_{A_{0 d}}^{1 / \alpha}\right)<$ 0 .

It follows after inspecting the solution (2.2), subject to (2.3)-(2.4), and Lemma 2.2 that the stability properties for arbitrary admissible initial conditions or admissible bounded controls are lost in general if $\alpha \geq 2$. However, it turns out that the boundedness of the solutions can be obtained by zeroing some of the functions of initial conditions. Note, in particular that $\varphi_{j}$ is required to be identically zero on its definition domain for $\overline{k-1} \cup\{0\} \ni$ $j<\alpha-1(\alpha \geq 2)$ in order that the $\Gamma$-functions will be positive (note that $\Gamma(x)$ is discontinuous at zero with an asymptote to $-\infty$ as $x \rightarrow 0^{-}$). This observation combined with Theorem 3.4 leads to the following direct result which is not a global stability result.

Theorem 3.5. Assume that $\alpha \geq 2$ and the constraint (3.27) holds with negative matrix measure $\mu_{2}\left(J_{A_{0 d}}^{1 / \alpha}\right)$. Assume also that $\varphi_{j}:[-h, 0] \rightarrow \mathbf{R}^{n}$ are any admissible functions of initial conditions for $\overline{k-1} \cup\{0\} \ni j \geq \alpha-1$ while they are identically zero if $\overline{k-1} \cup\{0\} \ni j<\alpha-1$. Then, the unforced solutions are uniformly bounded for all time independent of the delays. Also, the total solutions for admissible bounded controls are also bounded for all time independent of the delays. 


\section{Acknowledgments}

The author is grateful to the Spanish Ministry of Education for its partial support of this paper through Grant DPI2009-07197. He is also grateful to the Basque Government for its support through Grants IT378-10 and SAIOTEK S-PE09UN12.

\section{References}

[1] A. A. Kilbas, H. M. Srivastava, and J. J. Trujillo, Theory and Applications of Fractional Differential Equations, vol. 204 of North-Holland Mathematics Studies, Elsevier Science B.V., Amsterdam, The Netherlands, 2006.

[2] Z. Odibat, "Approximations of fractional integrals and Caputo fractional derivatives," Applied Mathematics and Computation, vol. 178, no. 2, pp. 527-533, 2006.

[3] Y. Luchko and R. Gorenflo, "An operational method for solving fractional differential equations with the Caputo derivatives," Acta Mathematica Vietnamica, vol. 24, no. 2, pp. 207-233, 1999.

[4] Y. F. Luchko and H. M. Srivastava, "The exact solution of certain differential equations of fractional order by using operational calculus," Computers $\mathcal{E}$ Mathematics with Applications, vol. 29, no. 8, pp. 73-85, 1995.

[5] R. C. Soni and D. Singh, "Certain fractional derivative formulae involving the product of a general class of polynomials and the multivariable H-function," Proceedings Indian Academy of Sciences. Mathematical Sciences, vol. 112, no. 4, pp. 551-562, 2002.

[6] R. K. Raina, "A note on the fractional derivatives of a general system of polynomials," Indian Journal of Pure and Applied Mathematics, vol. 16, no. 7, pp. 770-774, 1985.

[7] A. Babakhani and V. Daftardar-Gejji, "On calculus of local fractional derivatives," Journal of Mathematical Analysis and Applications, vol. 270, no. 1, pp. 66-79, 2002.

[8] A. Ashyralyev, "A note on fractional derivatives and fractional powers of operators," Journal of Mathematical Analysis and Applications, vol. 357, no. 1, pp. 232-236, 2009.

[9] B. Baeumer, M. M. Meerschaert, and J. Mortensen, "Space-time fractional derivative operators," Proceedings of the American Mathematical Society, vol. 133, no. 8, pp. 2273-2282, 2005.

[10] B. Ahmad and S. Sivasundaram, "On four-point nonlocal boundary value problems of nonlinear integro-differential equations of fractional order," Applied Mathematics and Computation, vol. 217, no. 2, pp. 480-487, 2010.

[11] F. Riewe, "Mechanics with fractional derivatives," Physical Review E, vol. 55, no. 3, part B, pp. 35813592, 1997.

[12] A. M. A. El- Sayed and M. Gaber, "On the finite Caputo and finite Riesz derivatives," Electronic Journal of Theoretic Physics, vol. 3, no. 12, pp. 81-95, 2006.

[13] R. Almeida, A. B. Malinowska, and D. F. M. Torres, "A fractional calculus of variations for multiple integrals with application to vibrating string," Journal of Mathematical Physics, vol. 51, no. 3, Article ID 033503, 12 pages, 2010.

[14] I. Schäfer and S. Kempfle, "Impulse responses of fractional damped systems," Nonlinear Dynamics, vol. 38, no. 1-4, pp. 61-68, 2004.

[15] F. J. Molz III, G. J. Fix III, and S. Lu, "A physical interpretation for the fractional derivative in Levy diffusion," Applied Mathematics Letters, vol. 15, no. 7, pp. 907-911, 2002.

[16] M. Ilic, I. W. Turner, F. Liu, and V. Anh, "Analytical and numerical solutions of a one-dimensional fractional-in-space diffusion equation in a composite medium," Applied Mathematics and Computation, vol. 216, no. 8, pp. 2248-2262, 2010.

[17] M. D. Ortigueira, "On the initial conditions in continuous-time fractional linear systems," Signal Processing, vol. 83, no. 11, pp. 2301-2309, 2003.

[18] J. Lancis, T. Szoplik, E. Tajahuerce, V. Climent, and M. Fernández-Alonso, "Fractional derivative Fourier plane filter for phase-change visualization," Applied Optics, vol. 36, no. 29, pp. 7461-7464, 1997.

[19] E. Scalas, R. Gorenflo, and F. Mainardi, "Fractional calculus and continuous-time finance," Physica A, vol. 284, no. 1-4, pp. 376-384, 2000.

[20] S. Das, Functional Fractional Calculus for System Identification and Controls, Springer, Berlin, Germany, 2008. 
[21] J. A. Tenreiro Machado, M. F. Silva, R. S. Barbosa et al., "Some applications of fractional calculus in engineering," Mathematical Problems in Engineering, vol. 2010, Article ID 639801, 34 pages, 2010.

[22] J. Sabatier, O. P. Agrawal, and J. A. Tenreiro Machad, Eds., Advances in Fractional Calculus: Theoretical Developments and Applications in Physics and Engineering, Springer, Dordrecht, The Netherlands, 2007.

[23] L. A. Zadeh and C. A. Desoer, Linear Systems Theory: The State Space Approach, McGraw- Hill, New York, NY, USA, 1963.

[24] M. De la Sen, "Application of the nonperiodic sampling to the identifiability and model matching problems in dynamic systems," International Journal of Systems Science, vol. 14, no. 4, pp. 367-383, 1983.

[25] M. De la Sen and N. Luo, "On the uniform exponential stability of a wide class of linear time-delay systems," Journal of Mathematical Analysis and Applications, vol. 289, no. 2, pp. 456-476, 2004.

[26] M. De la Sen, "Stability of impulsive time-varying systems and compactness of the operators mapping the input space into the state and output spaces," Journal of Mathematical Analysis and Applications, vol. 321, no. 2, pp. 621-650, 2006.

[27] J. Chen, D. M. Xu, and B. Shafai, "On sufficient conditions for stability independent of delay," IEEE Transactions on Automatic Control, vol. 40, no. 9, pp. 1675-1680, 1995.

[28] M. De la Sen, "On the reachability and controllability of positive linear time-invariant dynamic systems with internal and external incommensurate point delays," The Rocky Mountain Journal of Mathematics, vol. 40, no. 1, pp. 177-207, 2010.

[29] M. De la Sen, "A method for general design of positive real functions," IEEE Transactions on Circuits and Systems I, vol. 45, no. 7, pp. 764-769, 1998.

[30] M. De la Sen, "Preserving positive realness through discretization," Positivity, vol. 6, no. 1, pp. 31-45, 2002.

[31] M. De la Sen, “On positivity of singular regular linear time-delay time-invariant systems subject to multiple internal and external incommensurate point delays," Applied Mathematics and Computation, vol. 190, no. 1, pp. 382-401, 2007.

[32] P. N. Dutta and B. S. Choudhury, "A generalisation of contraction principle in metric spaces," Fixed Point Theory and Applications, vol. 2008, Article ID 406368, 8 pages, 2008.

[33] M. Arav, F. E. C. Santos, S. Reich, and A. J. Zaslavski, "A note on asymptotic contractions," Fixed Point Theory and Applications, vol. 2007, Article ID 39465, 6 pages, 2007.

[34] Y. J. Cho, S. M. Kang, and X. Qin, "Convergence theorems of fixed points for a finite family of nonexpansive mappings in Banach spaces," Fixed Point Theory and Applications, vol. 2008, Article ID 856145, 7 pages, 2008.

[35] S.-M. Jung and J. M. Rassias, "A fixed point approach to the stability of a functional equation of the spiral of Theodorus," Fixed Point Theory and Applications, vol. 2008, Article ID 945010, 7 pages, 2008.

[36] L. Cădariu and V. Radu, "Fixed point methods for the generalized stability of functional equations in a single variable," Fixed Point Theory and Applications, vol. 2008, Article ID 749392, 15 pages, 2008. 